\title{
On the Charge Storage and Decay Mechanism in Silicon Dioxide Electrets
}

\author{
W. Olthuis and P. Bergveld \\ MESA Research Institute, University of Twente, \\ Enschede, The Netherlands
}

\begin{abstract}
In this paper, a mechanism for both the storage and the decay of charge in a charged silicon dioxide layer is proposed. The oxide layer needs neutral electron traps to obtain stable trapped negative charge, after having been charged, resulting in an electret that can be applied in microphones. The deprotonization of the silanol groups, followed by charge injection, results in an electrochemical reaction with immobile $\mathrm{SiO}^{-}$as one of the reaction products. Decay of the stable charge thus obtained, can occur by the clustering of water molecules at inner silanol groups, resulting in a conductive hydrogen bonded network, which eventually leads to the discharge of the electret. Measurement results are presented, showing a considerable decrease in surface conductivity, after having grafted the $\mathrm{SiO}_{2}$ surface, resulting in covalently bonded, relatively long octadecyl dimethyl silane chains.
\end{abstract}

\section{INTRODUCTION}

$F^{\circ}$ Tor the conventional electret microphones fluorocarf bon polymers, such as Teflon-FEP ${ }^{\mathrm{TM}}$ and PTFE form an excellent electret material. The relatively thick polymer films form stable electrets and can relatively easy be deposited on piecewise manufactured microphones, having only moderately small dimensions.

The first generation of batch-processed microphones of which the backplate consists of micromachined silicon was also equipped with a polymer electret [1]. However, only after numerous tedious experiments was it possible to attach the wafer-scale Teflon film to the silicon backplate. Nevertheless, the relatively complicated batch processing of the essentially IC-incompatible polymer on the silicon wafers remained a problem.
At present, research is carried out on batch-processable microphones, manufactured completely with the standard steps of the silicon micromachining technology. This generation of microphones will no longer be equipped with polymer foil electrets: apart from the incompatibility of the deposition with standard silicon processing steps, as already mentioned, high temperature steps, not uncommon during micromachining techniques, would ruin the organic polymer. In addition, the sound-sensitive diaphragm of the new generation of microphones will also no longer consist of the formerly used $\mathrm{Mylar}^{\mathrm{TM}}$ membrane, but will be an IC-compatible membrane, such as $\mathrm{Si}_{3} \mathrm{~N}_{4}$. Consequently, the dimensions of the microphone are being redesigned, resulting in a smaller air gap width, in which the relatively thick Teflon polymer foil can no longer be used.

For the reasons mentioned above, the electret proper- 
ties of some inorganic dielectrics have been investigated, such as $\mathrm{Al}_{2} \mathrm{O}_{3}, \mathrm{Ta}_{2} \mathrm{O}_{5}, \mathrm{Si}_{3} \mathrm{~N}_{4}$ and $\mathrm{SiO}_{2}$. Although the results of experiments carried out with $\mathrm{Al}_{2} \mathrm{O}_{3}$ were promising, the main interest focuses on $\mathrm{SiO}_{2}$, because of its easy processing and compatibility with standard silicon technology as well as the fact that much is known in the literature about silica concerning its bulk properties (e.g., conductivity, maximum field strength) and interface phenomena, especially with respect to the interface with silicon.

Only a few papers report about charge retention on the $\mathrm{SiO}_{2}$-air interface, resulting in an external electric field [2-6]. Our previous investigations [7] led to the conclusion that an untreated $\mathrm{SiO}_{2}$ layer is a poor electret not because of its bulk conductivity, but due to its large lateral surface conduction. It has been shown, however, that such a $\mathrm{SiO}_{2}$ layer may be used as an excellent electret if the surface conductivity has been reduced by an appropriate chemical surface modification. This surface modification is adequately accomplished by a hydrophyllic to hydrophobic conversion using hexamethyldisilazane (HMDS).

Although it is known that HMDS substitutes to a certain amount the polar silanol groups on the surface of the silica by (apolar) trimethyl silyl groups [8], the exact effect this substitution has on the slowdown of the electret charge decay can only be described after we understand the charge trapping and decay mechanisms focused on the role of the surface and near-surface silanol groups.

Results of charge measurements, present at the $\mathrm{SiO}_{2}$ electrets that were prepared nearly 5 years ago, initiated investigations of the charge trapping mechanism and charge decay of $\mathrm{SiO}_{2}$ electrets.

The results of the investigations are in agreement with the behavior of another device where the outer $\mathrm{SiO}_{2}$ interface plays a major role: the ion sensitive field effect transistor (ISFET). The drift and hysteresis of this $\mathrm{pH}-$ sensitive device has been described using the same explanation as proposed in this paper for the charge trapping and decay of $\mathrm{SiO}_{2}$ electrets [9].

\section{TRAPPING AND DECAY OF CHARGE IN $\mathrm{SIO}_{2}$ LAYERS}

\subsection{CHARGE TRAPPING}

Dermanent storage of electronic charge in the oxide layer initially requires neutral electron traps (NET), which thus show a net negative charge of immobile nature, after being filled with electrons.

In order to obtain an electret microphone with sufficient sensitivity, the potential over the shallow air gap (typically 20 to $50 \mu \mathrm{m}$ ) between the electret and the diaphragm, which is induced by the fixed electret charge, must typically be -100 to $-300 \mathrm{~V}$. It is assumed, that the negative charge is trapped at the immediate proximity of the oxide-air interface. This assumption is based both on the discussion given below, and on the fact that $\mathrm{SiO}_{2}$ electrets of $1.1 \mu \mathrm{m}$ thickness $\boldsymbol{d}_{o x}$, have been charged to an equivalent electret voltage of $\sim-700 \mathrm{~V}$, which results in an electric field near the maximum field strength of $7 \mathrm{MV} / \mathrm{cm}$ found by others [10], only if the charge is considered as a sheet or thin layer of charge very near to the $\mathrm{SiO}_{2}$-air interface. In that case, the relation between the electret surface charge density $\sigma_{e}$ and the resulting equivalent electret potential $V_{e}$ is given by

$$
V_{e}=\frac{\sigma_{e} d_{o x}}{\epsilon_{o} \varepsilon_{r}}
$$

Typical values for a $d_{o x}=1.1 \mu \mathrm{m} \mathrm{SiO} 2$ layer $\left(\varepsilon_{r}=3.9\right)$ are $V_{e}=-150 \mathrm{~V}$ and, correspondingly, $\sigma_{e}=0.5 \mu \mathrm{C} / \mathrm{cm}^{2}$ or $\sim 3 \times 10^{12} e / \mathrm{cm}^{2}$.

The basic classification of charges and traps in $\mathrm{SiO}_{2}$ layers as posed for metal-SiO $\mathrm{S}_{2}-\mathrm{Si}$ (MOS) devices consists of 4 types [11]:

1. Si-SiO ${ }_{2}$ interface trapped charges $Q_{i t}$, due to the interruption of the periodic lattice structure. $Q_{i t}$ can exchange charges with silicon in a short time and can be as low as $10^{10} e / \mathrm{cm}^{2}$.

2. Immobile fixed oxide charge near the $\mathrm{Si}_{-}-\mathrm{SiO}_{2}$ interface $Q_{i t}$, which can not be charged or discharged easily. $Q_{i t}$ depends mainly on the processing conditions and the orientation of the silicon. This charge is thought to be caused by excess (trivalent) silicon or the loss of an electron from excess oxygen centers.

3. Mobile ionic charge $Q_{m}$, a space charge largely caused by alkali ions such as sodium. This oxide charge has its maximum concentration at the $\mathrm{Si}-\mathrm{SiO}_{2}$ interface and is mobile under bias-temperature aging conditions.

4. Oxide trapped space charges $Q_{o t}$, which can be created by radiation or electron injection and is considered to be associated with defects in the $\mathrm{SiO}_{2}$. $Q_{o t}$ consists of usually electrically neutral traps, which can be charged by introducing electrons (or holes) into the oxide. 
Of the four mentioned types of charge and traps, the first three are supposed to be unable to cause a stable electret, either because of the mobile nature of the charge and the position of the charge with respect to the $\mathrm{SiO}_{2}$ air interface or because of the fact that these charges do not form NET but appear as (mostly positively) charged centers instead of traps. Therefore, it may be possible that the charge, making up a stable electret, consists of filled NET of type $Q_{o t}$. It should be mentioned that $Q_{o t}$ is still a collection of traps of different cause and nature. Results of detailed investigations, described in the literature, however are, rather confusing: it is on the one hand reported, that annealing in $\mathrm{O}_{2}$ removes electron traps, which suggests oxygen deficiency as the cause of the trap centers [12], whereas on the other hand it is reported, that NET is generated by oxygen ion implantation [13]. The number of $\mathrm{NET} / \mathrm{cm}^{2}$ is in that case, however, too small to explain the electret behavior of $\mathrm{SiO}_{2}$.

Nicollian and coworkers assume a quite different origin of NET [14]. They suggest $Q_{o t}$ to be caused by the diffusion of water (at low temperature, $<200^{\circ} \mathrm{C}$ ) into the $\mathrm{SiO}_{2}$, resulting in NET formed by water-related impurities, which can capture an electron. This capturing mechanism, essentially an electrochemical reaction in which the NET is consumed, is accompanied by the evolution of neutral hydrogen. The result is a stable negatively charged center, which no longer traps charge. In the same paper, the authors also compared experimentally the spatial distribution of the negative charge with the water diffusion kinetics. It was found that both the charge and the water related centers were distributed over the first $40 \mathrm{~nm}$ of the oxide, at the oxide-air interface.

The following chain of reactions is proposed, resulting in stable trapped negative charge $[14,15]$. Formation of silanol groups, during or shortly after processing of the oxide (hydrolysis)

$$
\equiv \mathrm{Si}-\mathrm{O}-\mathrm{S} \equiv+\mathrm{H}_{2} \mathrm{O} \rightleftarrows 2 \equiv \mathrm{SiOH}
$$

The second reaction, at much lower temperatures $(<$ $200^{\circ} \mathrm{C}$ ) involves the deprotonization of the silanol groups by water

$$
\equiv \mathrm{SiOH}+\mathrm{H}_{2} \mathrm{O} \rightleftarrows \mathrm{SiO}^{-}+\mathrm{H}_{3} \mathrm{O}^{+}
$$

Clearly, the deprotonized silanol groups and the protonized water will form a closely adjacent pair. It is assumed, that an incoming electron can be captured by one of the fragments of this pair

$$
\mathrm{SiO}^{-}+\mathrm{H}_{3} \mathrm{O}^{+}+e \rightarrow \mathrm{SiO}^{-}+\mathrm{H}_{2} \mathrm{O}+\mathrm{H}
$$

This electrochemical reaction results in a stable negative charge with a negligible probability of being emitted after capturing; the trap is consumed and Coulomb repulsion keeps off other electrons.

By experiments, carried out with $\mathrm{SiO}_{2}$ hydrolyzed with tritiated water, it was shown, that indeed hydrogen evolution (Equation (4) occurs when an electron current flows through the hydrated $\mathrm{SiO}_{2}$ : the amount of hydrogen lost was found to have about a one-to-one correspondence to the amount of negative charge injected. From experiments both to create NET in an $\mathrm{H}_{2}$ ambient and to discharge samples by annealing them in an $\mathrm{H}_{2}$ ambient, it was concluded that atomic hydrogen is the immediate product of the capturing reaction rather than molecular hydrogen. This conclusion is in accordance with Equation (4).

The given explanation of charge trapping, mainly based on one paper [14], is supported by a later paper by $\mathrm{Di}$ Maria et al. [16], who also states that NET are associated with the presence of water related impurities: the deep trap sites are caused by $\mathrm{H}_{2} \mathrm{O}$ and $\mathrm{OH}$ bounded to $\mathrm{Si}$.

\subsection{CHARGE DECAY}

From the results of experiments, described in our previous paper [7] concerning $\mathrm{SiO}_{2}$ electrets, it was concluded that the cancellation of lateral surface conduction would result in prolonged charge retention. Because the silanol groups at a $\mathrm{SiO}_{2}$ surface were reported either to be the cause of the conductivity due to mobile protons of those groups or to attract, by their polar nature, physically absorbable water molecules, it was thought and shown that substitution of these surface groups sufficiently reduced the surface conductivity, according to the following mechanism.

Water reacts with the residual valences of the initially hydrophobic siloxane (SiOSi) surface, so that at ordinary temperature the surface becomes covered with hydrophyllic silanol (SiOH) groups

$$
\mathrm{Si}-\mathrm{O}-\mathrm{Si}+\mathrm{H}_{2} \mathrm{O} \rightleftarrows 2 \mathrm{SiOH}
$$

This process is called the chemisorption of water at the silica surface. These polar silanol groups may attract and bind water by physisorbtion

$$
\mathrm{SiOH}+\mathrm{H}_{2} \mathrm{O} \rightleftarrows \mathrm{SiOH}: \mathrm{OH}_{2}
$$

By substitution of the silanol groups with apolar trimethyl silyl groups of HMDS, neither the possible 
proton conduction of the silanol groups alone nor the formation of a monolayer of conductive water can occur

$2 \mathrm{SiOH}+\left(\mathrm{CH}_{3}\right)_{3} \mathrm{SiNHSi}\left(\mathrm{CH}_{3}\right)_{3} \rightarrow 2 \mathrm{SiOSi}\left(\mathrm{CH}_{3}\right)_{3}+\mathrm{NH}_{3}$

This silylation process might indeed reduce the number of surface silanol groups adequately, but will not reduce the number of inner, possibly even hydrated silanol groups, which fact forms a serious threat to the long-term charge stability, especially in humid environments.

It is reported that crystalline silica (quartz) gets 'coated' with a film of amorphous hydrolyzed silica with a thickness of $\sim 10$ to $\sim 40 \mathrm{~nm}$ [17]. This film can be etched with HF, but reappears in ambient relative humidity. Even if this layer of inner silanol groups itself might not cause conduction by internal protons, then there is still the problem of molecular water, physisorbed to the inner $\mathrm{SiOH}$ groups. The origin of this water can be twofold: first, the water molecules might diffuse into the already hydrolyzed silica film and react there, according to Equation (6, )and secondly, the water might be the product of the reported process of condensation [17] of the inner silanol groups, being Equation (5) in the opposite direction

$$
\mathrm{SiOH}+\mathrm{SiOH} \rightarrow \mathrm{SiOSi}+\mathrm{H}_{2} \mathrm{O}
$$

These internal water molecules can form a conductive hydrogen-bonded network, which might spread out in clusters, because energetically, these hydrogen bonded clusters of $\mathrm{H}_{2} \mathrm{O}$ molecules may be formed even before all the $\mathrm{SiOH}$ groups have adsorbed $\mathrm{H}_{2} \mathrm{O}$ molecules to form an uniform layer of $\mathrm{SiOH}: \mathrm{OH}_{2}$ groups [17].

Other experimental proof of the buried OH sites, which might either condense to water according to Equation (8 ), or may physically bind diffused water according to Equation (6), is reported in a paper by Bousse and Bergveld [9]. Beside the fast response of an ISFET, being a pure surface effect, the authors propose that the slow response of an ISFET to a $\mathrm{pH}$ step is due to the presence of a large number of $\mathrm{OH}$ sites buried beneath the surface. In addition, this slow response causes the observed hysteresis effect of the ISFET to a $\mathrm{pH}$ scan as well as the decreased sensitivity for higher $\mathrm{pH}$ values.

In conclusion, it is assumed that not only the conductivity of the surface but also that of the first $\sim 40 \mathrm{~nm}$ right under the silica-air interface, both caused by possibly hydrated silanol groups, play a decisive role in lateral conduction, causing charge decay of charged $\mathrm{SiO}_{2}$ electrets.

\section{EXPERIMENTAL}

$\mathrm{C}_{1 O_{2}}$ samples have been prepared from $5 \mathrm{~cm},<100>$ $\rho_{\mathrm{p}-\mathrm{Si}}, 5$ to $10 \Omega \mathrm{cm}$, previously cleaned $\mathrm{Si}$ wafers by wet thermal oxidation at $1150^{\circ} \mathrm{C}$ for $4 \mathrm{~h}$, resulting in a $\sim 1.1 \mu \mathrm{m}$ thick $\mathrm{SiO}_{2}$ layer. The oxide at the reverse side of the wafer was removed by chemical etching in order to allow to contact the samples during charging and measuring. The samples of which results are reported in this paper have been charged by the liquid-contact method. Other experiments, carried out with corona-charged samples however show, similar results. The equivalent electret voltage $V_{e}$ has been measured by a vibrating reed electrostatic voltmeter (Monroe isoprobe 244, accuracy better than $0.1 \%$, resolution $1 \mathrm{~V}$, lateral scan resolution $\sim 1 \mathrm{~mm}$ ). The samples, quarters of the $5 \mathrm{~cm}$ wafers, have been stored in small plastic boxes at 18 to $24^{\circ} \mathrm{C}$ and 40 to $70 \% \mathrm{RH}$.

A series of eight samples has been charged with different potentials, ranging from -200 to $-700 \mathrm{~V}$. The samples have been charged after the $\mathrm{SiO}_{2}$ surface had been treated with HMDS by liquid spinning, $4000 \mathrm{rpm}$ at $90^{\circ} \mathrm{C}$. Subsequently, the charged samples have been given a heat treatment for $50 \mathrm{~min}$ at $200^{\circ} \mathrm{C}$. This aging of the electrets prior to use, during which unstable traps are considered to be emptied, is a well-known pretreatment.

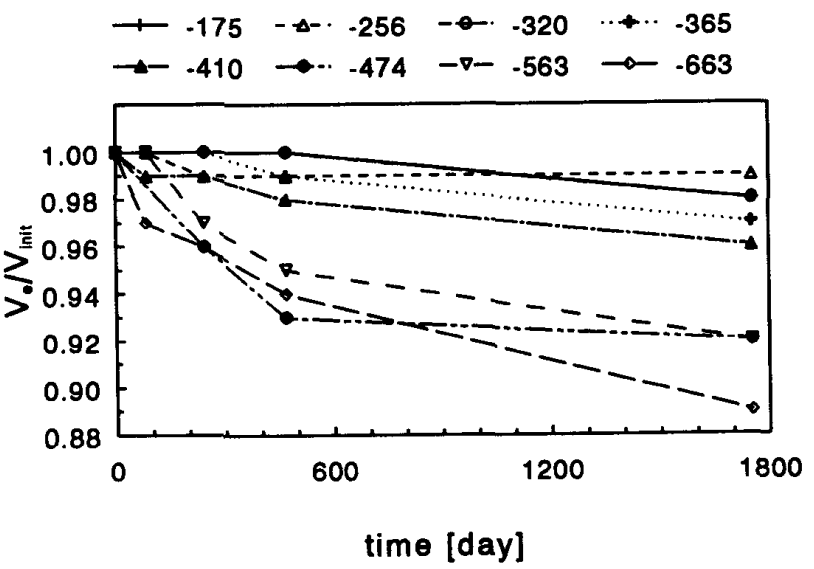

Figure 1.

Normalized charge decay at ambient laboratory conditions of $1.1 \mu \mathrm{m}$ thick $\mathrm{SiO}_{2}$ electrets, liquid contact charged, for different charge levels.

\section{RESULTS}

\subsection{HMDS TREATED SAMPLES}

THE series of 8 samples, prepared as mentioned in Sec1 tion 3 , has been charged $\sim 5$ years ago. Their equiv- 
alent electret potential $V_{e}$ has been measured from time to time and the intermediate measurement results have been reported earlier [7]. The results of the most recent measurements are included in Figure 1.

The potentials $V_{e}$ are normalized to their initial potential $V_{\text {init }}$ right after the thermal treatment, as mentioned in Section 3. The conclusions of our earlier paper [7] are consolidated by the recent measurements. The electric field of the electret, charged to $663 \mathrm{~V}$, amounts to $6 \mathrm{MV} / \mathrm{cm}$, which is very close to the reported theoretical maximum field strength of $7 \mathrm{MV} / \mathrm{cm}$ [10]. The life time of an electret is assumed to be equal to the time constant of the charge decay, i.e., the period in which the electret charge has decayed to $37 \%$ of its initial value [18]. In that case, the lower limit for the electret life time of the $663 \mathrm{~V}$ electret can be fixed at $40 \mathrm{y}$ instead of $20 \mathrm{y}$, formerly estimated [7]. The estimated life time of the $256 \mathrm{~V}$ electret of Figure 1 is even $\sim 500 \mathrm{y}$. The charge decay shows a clear dependence on the charge level and thus on the electric field in the oxide.

Apart from these positive results, there were some disturbing symptoms in relation to the homogeneity of the charged samples of several years old. Originally, the electrets have been very homogeneously charged $( \pm 2 \mathrm{~V})$ by the liquid contact method over an area of $>2 \mathrm{~cm}$. Recently, some of these samples show unexpected 'holes' in the charge of a few $\mathrm{mm}^{2}$, in which the charge sharply drops to $\sim 50$ to $70 \%$ of the original value.

This behavior, supported by other measurement results, was the motive for the investigation of the decay mechanism, as discussed in Section 2.2. It is assumed that in these electrets discharge occurs in patches around water molecules, bound at internal $\mathrm{SiOH}$ groups, forming a local conductive hydrogen bonded network. The gradual spreading of these internal water molecules might eventually cause the total discharge of the electret.

The number of electrons, necessary for a typical application in a microphone, has been calculated in Section 2.1 and is $\sim 3 \times 10^{12} \mathrm{e} / \mathrm{cm}^{2}$. The reported number [17] of silanol groups at the $\mathrm{SiO}_{2}$ surface, however, is $5 \times 10^{14} \mathrm{SiOH} / \mathrm{cm}^{2}$. Therefore, if the number of electrons per silanol group is restricted to the surface groups, then only one electron per 167 silanol groups is needed to obtain the necessary amount of charge in the $1.1 \mu \mathrm{m}$ thick electret.

It is, however, reported [8], that only $\sim 95 \%$ of the surface silanol groups is substituted by trimethyl silyl groups after an HMDS treatment. This percentage is based on the decrease of the surface conductivity. Because this decrease is not only the result of a reduced number of silanol groups, but also of a decreased number of physisorbed water molecules at these silanol groups, the percentage of substituted silanol groups will even be lower; due to steric hindrance of the trimethyl silane, not all silanol groups can be substituted by these groups [19]. Therefore, it is concluded that even after the HMDS treatment, the remaining number of uncharged surface silanol groups may either cause discharge of the relatively small number of electrons or form paths for the diffusing $\mathrm{H}_{2} \mathrm{O}$ molecules to the internal $\mathrm{OH}$ groups, causing cluster formation of water. As soon as the clusters contact each other, this may lead to the discharge of the electret.

Comparing Sections 2.1 and 2.2 concerning the trapping of charge and the decay of charge due to local conduction, respectively, in relation to the experimental results, it has to be stated that a dilemma shows up: silanol groups are considered to be necessary for the stable trapping of charge, whereas silanol groups also may cause, eventually, the unexpected end to the electret life. The charge traps however, are assumed to be generated during the oxidation process and are possibly located deeper into the oxide than the conductive sites, which may appear additionally at a later point of time. It will be obvious that if later formation of surface and buried sites under the influence of ambient water molecules should be prevented, a more definite covering of the oxide is necessary. Therefore, new silylating agents, which might effectively close the surface of the $\mathrm{SiO}_{2}$ for water molecules are tested and the first results are presented in the next Section.

\subsection{TREATMENT WITH OCTADECYL DIMETHYL (DIMETHYLAMINO) SILANE}

Silicon wafers, oxidized in the way described in Section 3, have been covalently grafted with monolayers of octadecyl dimethyl silane in order to obtain a dense, hydrophyllic surface. This grafting process and the subsequent chromatographic measurement to determine the density of the grafted chains have been carried out by Martelet and coworkers [20], The details of the preparation of octadecyl dimethyl (dimethylamino) silane, the grafting process and the density measurements are reported in one of their papers [20].

HMDS and octadecyl dimethyl (dimethylamino) silane applied on $\mathrm{a} \mathrm{SiO}_{2}$ surface are quite similar with respect to the nature of the bonding (covalent), the bonding energy and, roughly, the number of substituted silanol groups. However, the latter of the two proposed treatments is presumed to show a better coverage due to stearic packing of the grafted alkyl chains. The strong hydrophobic 
repulsion of the hydrocarbon tails from water results in a tightly packed and therefore impermeable coating [21].

To investigate the expected decrease in surface conductivity after the grafting process of the samples, a preliminary experiment was carried out. Several treated and untreated $\mathrm{SiO}_{2}$ samples have been locally corona charged through a $5 \mathrm{~mm}$ wide slit in a grounded one-side metalized printed circuit board, placed on top of the oxide sample.

The measurement results of a lateral scan over the partly charged sample, perpendicular to the small profile of charge, are shown in Figure 2. In Figure 2(a), the decay of the applied charge profile on an unmodified $\mathrm{SiO}_{2}$ sample due to lateral surface conductivity is clearly visible, whereas the charge profile of the grafted $\mathrm{SiO}_{2}$ sample, shown in Figure 2(b), remains virtually unchanged under the same conditions.

These preliminary results show a considerable decrease in surface conductivity after grafting of the $\mathrm{SiO}_{2}$ samples, stored under room conditions. Further experiments must clarify whether this promising effect remains under less favorable conditions of storage, such as a very high relative humidity. A comparison of both octadecyl dimethyl (dimethylamino) silane and HMDS treated $\mathrm{SiO}_{2}$ samples stored under these conditions should be carried out.

\section{CONCLUSIONS}

THE monopolar charge in a $\mathrm{SiO}_{2}$ electret is assumed 1 to be caused by deprotonization of silanol groups, followed by charge injection, resulting in an electrochemical reaction with immobile $\mathrm{SiO}^{-}$as one of the reaction products.

Not only the conductivity of the $\mathrm{SiO}_{2}$ surface, but also that of the first $\sim 40 \mathrm{~nm}$ directly under the silica-air interface, both caused by possibly hydrated silanol groups, play a decisive role in lateral conduction, causing charge decay of $\mathrm{SiO}_{2}$ electrets.

The estimated life time of $\mathrm{SiO}_{2}$ electrets, treated with HMDS and stored under room ambient conditions, charged to a corresponding internal electric field from 1 to $6 \mathrm{MV} / \mathrm{cm}$ ranges from 40 to $500 \mathrm{y}$, based on experimental results.

Charge decay under severe storage conditions (in particular high relative humidity) can possibly be prevented by effectively closing the surface of $\mathrm{SiO}_{2}$ for water molecules with monolayers of dense, covalently bonded, relatively long alkyl chains.
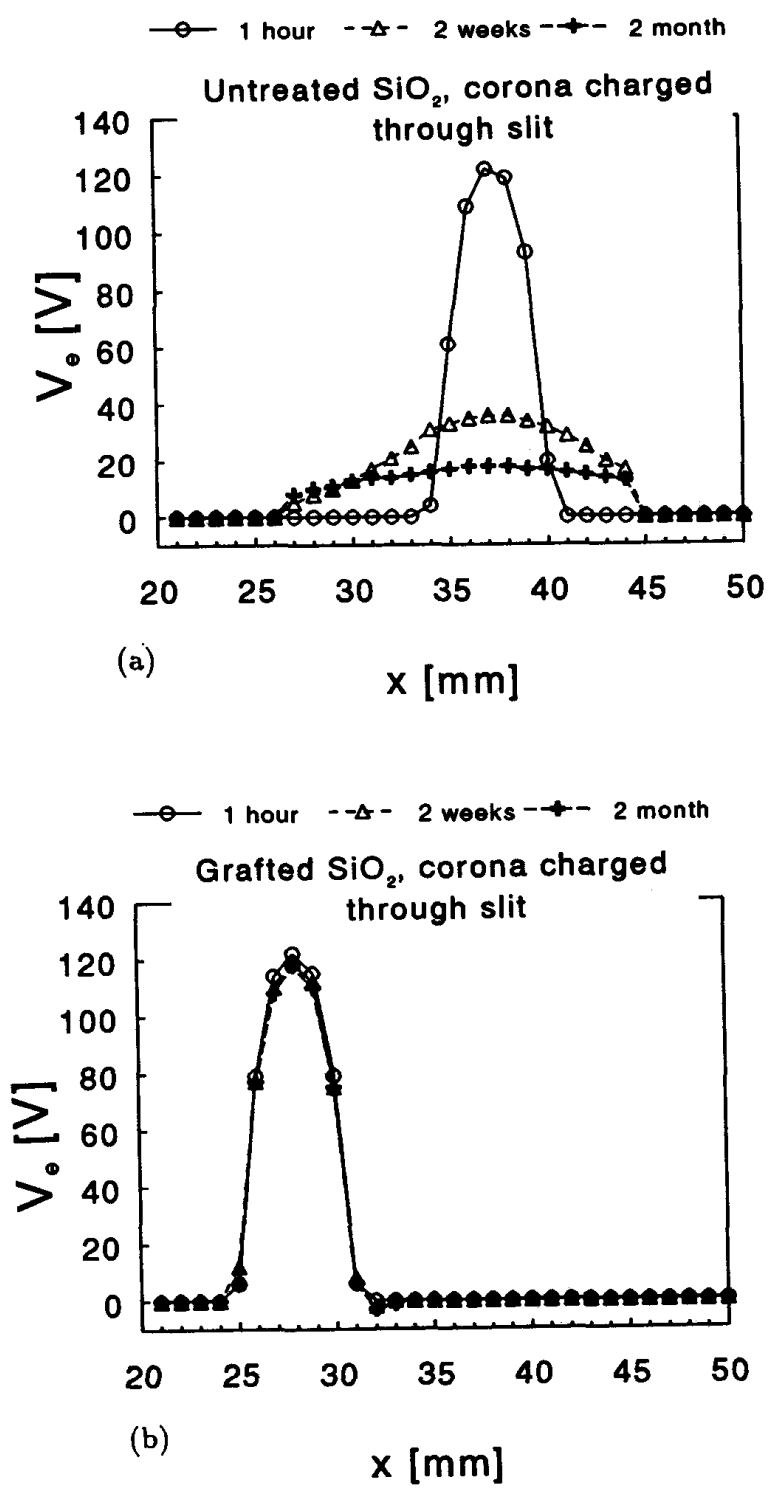

Figure 2.

The measured results of a lateral scan of the potential, perpendicular to the $5 \mathrm{~mm}$ wide charge profile. The potential has been measured $1 \mathrm{~h}, 0.5$ and 2 month after initial charging. (a) Untreated $\mathrm{SiO}_{2}$ sample, (b) octadecyl dimethyl (dimethylamino) silane treated $\mathrm{SiO}_{2}$ sample.

\section{ACKNOWLEDGMENT}

The authors wish to thank Dr. C. Martelet (Laboratoire de Physicochimie des Interfaces, Ecole Centrale de Lyon) for grafting the $\mathrm{SiO}_{2}$ samples. The discussions 
with R. E. G. van Hal during the preparation of this paper have been very helpful.

\section{REFERENCES}

[1] A. J. Sprenkels, A silicon subminiature electret microphone, Thesis, Twente University, ISBN 90-900 2052-7, 1988.

[2] A. E. Guile, "Stored Charges in Relative Thin Oxide Films", J. Phys. D. Appl. Phys., Vol. 5, pp. 11531156, 1972.

[3] G. M. Sessler and J. E. West, "Charge Properties of Electron-Beam Irradiated Dielectrics", in Electrets, Charge Storage and Transport in Dielectrics, ed. M. M. Perlman, The Electrochem. Soc., Princeton, pp. 292-299, 1973.

[4] D. Hohm and R. Gerhard-Mulhaupt, "SiliconDioxide Electret Transducer", J. Acoust. Soc. Am., Vol. 75 , pp. $1297-1298,1984$.

[5] P. Günther, "Charging, Long-Term Stability, and TSD Measurements of $\mathrm{SiO}_{2}$ Electrets", IEEE Trans. Electric. Insul., Vol. 24, pp. 439-442, 1989.

[6] P. Günther, "Mechanism of Charge Storage in Electron-Beam or Corona-Charged Silicon-Dioxide Electrets", IEEE Trans. Electric. Insul., Vol. 26, pp. 42-48, 1991.

[7] J. A. Voorthuyzen, W. Olthuis, P. Bergveld and A. J. Sprenkels, "Research and Development of Miniaturized Electrets", IEEE Trans. Electric. Insul., Vol. 24, pp. 255-266, 1989.

[8] J. A. Voorthuyzen, K. Keskin and P. Bergveld, "Investigations of the Surface Conductivity of Silicon Dioxide and Methods to Reduce It", Surface Sci. Vol. 187, pp. 201-211, 1987.

[9] L. Bousse and P. Bergveld, "The Role of Buried OH Sites in the Response Mechanism of Inorganic-Gate pH-Sensitive ISFET", Sensors and Actuators, Vol. 6, pp. $65-78,1984$.

[10] R. Williams and H. Woods, "High Electric Fields in Silicon Dioxide Produced by Corona Charging", J. Appl. Phys., Vol. 44, pp. 1026-1028, 1973.
[11] S. M. Sze, Physics of Semiconductor Devices, 2nd edition, Wiley \& sons, New York, 1981.

[12] M. Aslam and P. Balk, "Processing Dependence and Structure of Hole Traps in $\mathrm{SiO}_{2}$ ", Abstr. Int. Conf. Insul. Films on Semiconductors, Eindhoven, p. 22, 1983.

[13] C. T. Sune, A. Reisman and C. K. Williams, "Oxygen Ion Defect Generation in Insulated-Gate FieldEffect Transistors and X-Radiation Susceptibility of Ion-Damaged Gate Insulators", J. Electrochem. Soc., Vol. 137, pp. 3574-3578, 1990.

[14] E. H. Nicollian, C. N. Berglund, P. F. Schmidt and J. M. Andrews, "Electrochemical Charging of Thermal $\mathrm{SiO}_{2}$ Films by Injected Electron Currents", J. Appl. Phys., Vol. 42, pp. 5654-5664, 1971.

[15] D. L. Wolters, "The Role of Water in the Oxidation of Silicon", Proc. Conf. Insul. Films on Semiconductors, Conference Series no. 50, G. G. Roberts and M. J. Morant, ed., Durham, pp. 18-27, 1979.

[16] D. J. DiMaria, D. W. Dong, C. Falcony, T. N. Theis, J. R. Kirtley, J. C. Tsang, D. R. Young, F. L. Pesavento and S. D. Brorson, "Charge Transport and Trapping Phenomena in Off-Stoichiometric Silicon Oxide Films", J. Appl. Phys., Vol. 54, pp. 5801$5827,1983$.

[17] R. K. Iler, The chemistry of Silica, Wiley \& Sons, New York, 1979.

[18] A. J. Sprenkels, W. Olthuis and P. Bergveld, "The Application of Silicon Dioxide as an Electret Material", Proc. 6th Int. Symp. on Electrets, ISE 6, D. K. Das-Gupta and A. W. Pattullo, ed., Oxford, pp. 165-169, 1988.

[19] A. C. Zettlemoyer and H. H. Hsing, "Water on Organosilane-Treated Silica Surfaces", J. Colloid Interface. Sci., Vol. 58, pp. 263-274, 1977.

[20] Y. Duvault, A. Gagnaire, F. Gardies, N. JaffrezicRenault, C. Martelet, D. Morel, J. Serpinet, J. L. Duvault, "Physicochemical Characterization of Covalently Bonded Alkyl Monolayers on Silica Surfaces", Thin Solid Films, Vol. 185, pp. 169-179, 1990.

[21] H. O. Finklea, S. Avery, M. Lynch and T. Furtsch, "Blocking Oriented Monolayers of Alkyl Mercaptans on Gold Electrodes", Langmuir, Vol. 3, No. 3, pp. 409-413, 1987.

This manuscript is based on a paper given at the 7th International Symposium on Electrets, Berlin, Germany, 25-27 September 1991.

Manuscript was received on 25 March 1992 